Final Technical Report

On the Interagency Agreement No. DE-AI02-95ER54293

\title{
Determination of Atomic Data Pertinent to the Fusion Energy Program
}

J. Reader, Principal Investigator

11 June 2013 


\section{Introduction}

In the sections below we summarize progress that has been made on the determination of atomic data pertinent to the fusion energy program since the start of the present contract period.

In addition to the various technical accomplishments reviewed, the NIST group has contributed in a number of ways to the U.S. participation in the international effort to advance fusion energy science.

The NIST Atomic Spectroscopy Data Center continues to be a key contributor to the IAEA Atomic and Molecular Data Center Network. We participated in the $20^{\text {th }}$ Meeting of the Network in September 2009 at the IAEA headquarters in Vienna and delivered a progress report of our data assessment and compilation activities. W. L. Wiese also participated in a Coordinated Research Project of the IAEA on "Atomic Data for Heavy Element Impurities in Fusion Reactors" and reported on NIST research that produced new spectroscopic data and critical compilations for heavy elements. $\mathrm{He}$ reported on a compilation for Fe I and II and work on highly ionized tungsten spectra with the NIST EBIT at the final research coordination meeting, which took place at the IAEA Headquarters in March 2009. Furthermore, we actively participate in the IAEA Code Center Network since its formation in 2005 . Yu. Ralchenko attended the $3^{\text {rd }}$ meeting of the CCN in October 2010 and gave a talk on NIST codes for fusion research. Also our group was invited to be a member of the IAEA Coordinated Research Project "Spectroscopic and Collisional Data for Tungsten from $1 \mathrm{eV}$ to $20 \mathrm{keV}$ " which will be initiated in December 2010.

For the last several years, NIST has taken a leading role in organizing the Non-Local Thermodynamic Equilibrium (NLTE) Code Comparison Workshops that enable fusion researchers to test different approaches and models in plasma population kinetics. These Workshops have become a primary venue for plasma physicists using collisionalradiative (CR) modeling for calculation of plasma emission, ionization balance, radiative power losses, and other critical parameters for operation of various plasma devices. The benchmark numerical data calculated by the Workshop participants are currently available for online access at the NIST website with extensive options for data selection, manipulation, and presentation. These data are accessed on a daily basis by many researchers from the U.S. and other countries.

The NIST website also contains an online collisional-radiative code that allows real-time population kinetics simulations for arbitrary plasma conditions. This code, named FLYCHK and developed primarily at Lawrence Livermore National Laboratory, has numerous options to include a radiation field, a non-Maxwellian electron energy distribution function, opacity, and other important effects. As of October 2010, about 350 scientists from various plasma laboratories and research groups routinely perform simulations with FLYCHK for magnetic fusion research, laser-produced plasmas, astrophysics, and other fields of physics. 
NIST has recently developed a more detailed collisional-radiative code (NOMAD), which has been successfully used to interpret plasma emission from tokomaks, EBITs, and other fusion-related devices. It is currently being used to advance the state of Charge Exchange Recombination Spectroscopy and Motional Stark Effect, both important diagnostic tools for tokamak plasmas. NIST will make this code available on its website for use by registered users. A primary attribute of both FLYCHK and NOMAD is their seamless utilization of the atomic data available in the NIST Atomic Spectra Database.

Finally, NIST has been leading an international group whose goal is to develop new internet protocol standards for the exchange of large volumes of diverse atomic data. These new standards are necessary for full utilization of the large volumes of data that are now available to researchers through venues like our Atomic Spectra Database.

A. Kramida gave an invited paper on "Recent progress in the spectroscopy of tungsten" at the 10th International Colloquium on Atomic Spectra and Oscillator Strengths for Astrophysical and Laboratory Plasmas, Berkeley, California, August 2010. Kramida also gave an invited paper on "Recent Developments in the NIST Atomic Databases" at ICAMDATA 7, the International Conference on Atomic and Molecular Data and Their Applications, Vilnius, Lithuania, September 2010. J. Reader gave invited talks at the Institute of Spectroscopy in Moscow on "The Strange and Emotional Story of Mo VI," and "Spectroscopy and Atomic Physics of Highly Charged Heavy Elements Observed with an Electron Beam Ion Trap at NIST," September 2010. Yu. Ralchenko gave invited talks on "XML Schema for Atoms, Molecules and Solids" at the IAEA Technical Meeting at NIFS, Japan, March 2010, on "Modeling of Hot Plasmas with Particle Beams and Comparison of Collisional Radiative Codes" at the Atlantic Centre for Atomistic Modelling (ACAM) Workshop on Plasma Modeling at the University College Dublin, Ireland, April 2010, on "Introduction to XML Schema for Atoms, Molecules, and Solids (XSAMS)" at the Virtual Atomic and Molecular Data Center (VAMDC) Annual Meeting, Milton Keynes, UK, April 2010, on "EUV Spectroscopy of Highly-Charged High-Z Ions on the NIST EBIT" at Fortschungzentrum Juelich, Germany, April 2010, on "NIST Codes for Fusion Research" at the Code Center Network Meeting at IAEA, Vienna, Austria, September 2010, on "Report on the NLTE-6 Workshop" at the Workshop on Radiative Properties of Hot Dense Matter, Marbella, Spain, October 2010. J. J. Curry gave an invited talk on 'Spectroscopic Research Projects at NIST on Light Elements' at the IAEA Meeting on Light Element Atom, Molecule and Radical Behavior in the Divertor and Edge Plasma Regions, November 2009. 


\section{Identification of Spectral Lines of Impurity Ions (J. Reader)}

Work has continued on studies of spectra of moderately charged atoms for the diagnostics of first wall and divertor regions of tokamaks. Our work on the one-electron spectrum of molybdenum, Mo VI was published in a special issue of Journal of Physics B, that appeared in April 2010. Our colleague Tauheed Ahmad from India visited during the last three months of 2009 and furthered our progress on the spectrum of Mo V. A first draft of a manuscript was completed. Work will be continuing as possible to complete the manuscript.

Our work on the spectrum of three-times ionized cerium, $\mathrm{Ce}^{3+}$, was completed and published in the Physical Review. Our work on extending the analysis of doubly ionized magnesium was also completed and published in.Physica Scripta.

Together with collaborators from the Naval Research Laboratory, we used the NIST grazing incidence spectrograph to make new observations of moderately ionized iron, $\mathrm{Fe}$ VIII- Fe X. The data are currently being analyzed.

\section{Publications}

1. J. Reader, "Spectrum and Energy Levels of Five-Times Ionized Molybdenum (Mo VI)," J. Phys. B: At. Mol. Opt. Phys. 43, 074024 (2010)..

2. J. Reader and J.-F. Wyart, "Observation of Inner-Shell-Excited Configurations in Triply Ionized Cerium, Ce ${ }^{3+}$," Phys. Rev. A 80, 042517 (2009).

3. C. M. Brown, A. E. Kramida, U. Feldman, and J. Reader, "Extension of the resonance line series of Mg III," Physica Scripta, 80, 065302 (2010).

\section{Talks}

1. "Spectrum and Energy Levels of Triply-Ionized Cerium $\left(\mathrm{Ce}^{3+}\right)$," J. Reader and J.-F. Wyart, $16^{\text {th }}$ International Conference on Atomic Processes in Plasmas, Monterey, California, March 2009.

2. "The Strange and Emotional Story of Mo VI," J. Reader, Insitute of Spectroscopy, Moscow, September 2010.

3. "Magnetic-Dipole Lines in Highly-Charged Ions of Tungsten," J. Reader, DAMOP2010, Houston, Texas, May 2010.

\section{Spectroscopic Data Assessment and Compilations (W. Wiese and J. Reader)}

Our work continues to produce critical compilations of the energy levels and observed 
spectral lines of the noble gases. Previously, we published compilations for all stages of ionization of krypton and xenon and for neutral neon. Our extensive compilation for Ar II - Ar XVIII was published in 2010.

Our newly revised compilation of spectra of highly ionized tungsten was published in the Canadian Journal of Physics. Other compilations for numerous elements are listed below.

Our critical compilations of atomic transition probabilities (or radiative transition rates) continued for a number of atoms and ions relevant to fusion research, for light as well as heavy elements. In all these compilations, we greatly improved the earlier NIST data tables in both quantity and quality.

For the lightest atoms - hydrogen, deuterium, and tritium as well as helium and lithium with their ions - we published a comprehensive compilation of all experimental data on observed fine and hyperfine structure.

For beryllium and boron, we published a greatly expanded compilation in 2010. This was especially timely, since according to current plans beryllium is expected to be the first wall material in the main chamber of ITER. The new tabulation for the transition probabilities of Be I contains 240 lines, compared to only 26 lines in the previous NIST compilation. Also, the great majority of our new $\mathrm{Be} I$ data are estimated to be accurate within $10 \%$, while in earlier NIST ASD editions most are listed as uncertain by either $25 \%$ or $50 \%$.

A compilation of wavelengths, energy levels, and transition probabilities for all spectra of cesium has been completed and published. Spectroscopic data of barium from doubly ionized to hydrogen-like was published in the Journal of Physical and Chemical Reference data. A compilation of data for neutral strontium has been completed and is being prepared for publication. Compilations for several ions of neon and fluorine are in progress.

\section{Publications}

1. E. B. Saloman, "Energy Levels and Observed Spectral Lines of Ionized Argon, Ar II through Ar XVIII," J. Phys. Chem. Ref. Data 39, 033101 (2010).

2. A. E. Kramida, "Recent progress in Spectroscopy of Tungsten", in press (Can. J. Phys., 2010).

3. A. E. Kramida, "A Critical Compilation of Experimental Data on Spectral Lines and Energy Levels of Hydrogen, Deuterium, and Tritium," At. Data Nucl. Data Tables, 96, $586(2010)$.

4. J. R. Fuhr and W. L. Wiese, "Tables of atomic transition probabilities for beryllium and boron," J. Phys. Chem. Ref. Data 39, 013101 (2010).

5. J. E. Sansonetti, "Wavelengths, Transition Probabilities, and Energy Levels for the Spectra of Cesium (Cs I-Cs LV)," J. Phys. Chem. Ref. Data 38, 761 (2009).

6. J. E. Sansonetti, "Wavelengths, Transition Probabilities, and Energy Levels for the 
Spectra of Highly Ionized Barium (Ba III-Ba LVI)," in press (J. Phys. Chem. Ref. Data, 2010).

Talks

Numerous talks were presented at recent ICAMDATA, ASOS, DAMOP, and IAEA meetings.

\section{Expansion and Upgrade of the NIST Atomic Databases (Yu. Ralchenko and A.E. Kramida)}

The new version of the NIST Atomic Spectra Database (ASD), v.4.0.0 was released for online access in September 2010. While minor improvements and corrections were added, and a great amount of new data was included, the main feature of the new release consists of seamless integration with the NIST Atomic Spectra Bibliography Databases on atomic energy levels and transition probabilities. Another important addition is the addition of data on atomic isotopes. Since April 2008 (version 3.1.5) the following data additions and corrections were made:

1. New high-precision data sets of energy levels and spectral lines were added/updated for hydrogen, deuterium, and tritium. This replaces the old hydrogen data that came from outdated theoretical calculations.

2. In He I, all energy levels were updated with more precise values from recently published data. The line list was extended from 351 to 2300 lines. Most of them include high-precision transition probability data. The He II line list now includes 140 fine-structure transitions, all with transition probability values and improved energy levels precision. In addition to the most abundant isotope ${ }^{4} \mathrm{He}$, we now included energy levels data for the isotope ${ }^{3} \mathrm{He}$.

3. Improved and extended transition probability data from recent NIST compilations were added to the Li I-III, Be I-IV, B I-V, C I-II, and N I-II line lists. New data tables were added for neutral and singly ionized isotopes ${ }^{10,11} \mathrm{~B}$.

4. Energy levels and lines are included for all spectra of tungsten, neutral $W$ through $\mathrm{W}^{73+}$.

5. Energy levels and lines were added/updated for all spectra of sodium (Na I through $\mathrm{Na} \mathrm{XI}$ ) and potassium (K I through $\mathrm{K} \mathrm{XIX}$ ). New data tables were added for neutral isotopes ${ }^{39,40,41} \mathrm{~K}$.

6. Energy levels, observed lines, and transition probability data lists for $\mathrm{Fe} I$ were upgraded with extensive precise Fourier transform spectroscopy data and with revised transition probability data from a recent NIST compilation. The new line list of Fe I contains more than 10,000 lines.

7. Transition probability data for Fe II were updated from a recent NIST compilation. The new line list of Fe II contains more than 1000 lines with precise wavelengths and improved transition probabilities.

8. Data tables for energy levels and spectral lines of $\mathrm{Hg}$ I were updated from a recent NIST compilation. New data tables for energy levels and spectral lines of the neutral 
isotope ${ }^{198} \mathrm{Hg}$ were added.

9. Data tables for energy levels of Ag I-II, Au I-II, Ac I-II, and Bi I-II were added.

As of October 2010, NIST ASD includes 168,000 spectral lines and 80,000 energy levels.

The NIST Atomic Bibliographic Databases are now being updated regularly, typically every two weeks. They have been upgraded to new versions in September of 2010. As of October 12, 2010, the Atomic Transition Probability Bibliographic Database (version 9.0) contains 8338 references, dating from 1914 through 2010; the Bibliographic Database on Atomic Spectral Line Broadening v.3.0 contains 6461 references from 1889 through 2010; and the Atomic Energy Levels and Spectra Bibliographic Database v.2.0 contains 15,334 references from 1802 through 2010. An invited talk about the development of atomic-physics databases at NIST was presented at the ICAMDATA meeting in Lithuania in September 2010, and a poster on the development of the bibliography databases was presented at the DAMOP meeting in Houston, Texas in May 2010.

We continued development of the online collisional-radiative code FLYCHK that provides unique capabilities for simulation of plasma emission and kinetics under arbitrary conditions. FLYCHK is currently being used by almost 270 researchers from the USA and other countries.

We continued taking part in the international effort to develop the new standard format for atomic data exchange over the Internet. The first version of the new standard, XML Schema for Atoms, Molecules and Solids (XSAMS) v. 0.1, was released in September 2009. The web page of XSAMS at http://www-amdis.iaea.org/xsams/ contains project description, the schema file(s), documentation and contact information. We also collaborate with the Virtual Atomic and Molecular Data Center project on implementation of the XSAMS standards.

\section{Publications}

1. Yu. Ralchenko, R.E.H. Clark, M.L. Dubernet, S. Gagarin, D. Humbert, P.A. Loboda, N. Moreau, E. Roueff and D.R. Schultz, "Development of New Standards for Exchange of Atomic and Molecular Data," in: $6^{\text {th }}$ International Conference on Atomic and Molecular Data and Their Applications, AIP Conference Proceedings 1125, 207 (2009).

2. Yu. Ralchenko, "Online Databases and Computational Tools for non-LTE Spectroscopy," Physica Scripta T 134, 014025 (2009).

3. M.-L. Dubernet et al, "Virtual Atomic and Molecular Data Centre," J. Quant. Spectr. Rad. Transfer 111, 2151 (2010).

Talks 
1. "Recent Developments in the NIST Atomic Databases", International Conference on Atomic and Molecular Data and Their Applications, Vilnius, September 2010 (A. E. Kramida).

2. "XSAMS", LAEA Technical Meeting, NIFS, Japan, March 2010.

3. "Introduction to XSAMS", VAMDC Annual Meeting, Milton Keynes, UK, April 2010 (Yu. Ralchenko)

4. "New Additions to XSAMS," VAMDC Technical Meeting, Paris, France, July 2010 (Yu. Ralchenko)

5. "NIST Codes for Fusion Research", IAEA Code Center Network Meeting, Vienna, Austria, September 2010 (Yu. Ralchenko).

\section{Collision and Spectroscopy Experiments with Highly Charged Ions on EBIT (John Gillaspy)}

We carried out several measurement campaigns with the NIST EBIT, acquiring approximately 700 spectra from 10 different elements (W, $\mathrm{Au}, \mathrm{Ta}, \mathrm{Hf}, \mathrm{Xe}, \mathrm{Kr}, \mathrm{Fe}, \mathrm{Ar}$, $\mathrm{Ne}, \mathrm{N}$ ), in three different spectral ranges (two in the extreme ultraviolet and one in the $\mathrm{x}$ ray), under a wide variety of conditions (different electron collision energies, current densities, background pressures, trapping times, etc.). Well-known lines from the lighter elements provided calibrations for the previously unknown lines in the heavier elements. As a result of this work, we have supplemented the heavy-element data we took last year (extending it to the range $1.84 \mathrm{keV}$ to $5.15 \mathrm{keV}$ for the case of $\mathrm{Hf}, \mathrm{Ta}$, and $\mathrm{Au}$, for example) and we have improved the accuracy of our calibration. A paper on this was completed and submitted to J. Phys. B. The paper will report the identification of over 150 lines, from Rb-like $\mathrm{Ha}$ (charge $35+$ ) to Co-like $\mathrm{Au}$ (charge $52+$ ). Over $75 \%$ of these lines are entirely new (not found in the literature). A second manuscript which presents additional data taken this year on $\mathrm{W}$ is nearing completion (results were recently presented at an international conference).

We have completed the analysis of the photoionization data ( $>150 \mathrm{x}$-ray spectra) that we recorded last year when we helped deploy an EBIT at the Advanced Photon Source at the Argonne National Laboratory. This work involved $\mathrm{Kr}$ and $\mathrm{Ar}$ ions, as a prelude to studying heavier elements. The signal-to-noise ratio was very low, but when averaged showed a peak at the predicted wavelength and intensity--the first laboratory evidence of hard x-ray photoionization of a highly charged ion. We submitted this for publication and also detailed future improvements in the apparatus which should lead to several orders of magnitude improvement in the signal-to-noise ratio. This work opens the door to a wide range of measurements of hard x-ray photoionization cross sections for highly charged ions (from which one can also obtain radiative recombination cross sections directly via the Milne relation), an area for which there is virtually no prior experimental 
atomic data available.

We completed our analysis of Fe XVII x-ray data and submitted a paper that details the use of two line intensity ratios for reliable plasma diagnostics. This paper includes new theoretical work from three other groups outside of NIST, and resolves large (50\%) discrepancies previously reported in the literature. Agreement is now typically $10 \%$ or less, which is within the estimated uncertainties. The situation was complicated because the discrepancies arose for multiple reasons (some in the theory of excitation cross sections, some in the experimental line ratios, and some in the theory of photoionization cross sections that were used to normalize one of the experiments performed elsewhere). This publication opens the door for trustworthy application of Fe XVII for precision plasma diagnostics.

In order to extract more scientific value from the data we published the $\mathrm{D}$-lines of $\mathrm{Na}$-like ions of $\mathrm{W}$ and similar mass elements [Gillaspy et al., Phys. Rev. A, 80, 010501(R), 2009], we have obtained highly accurate $a b$ initio transition energy predictions and compared them to our results, allowing us to obtain the most accurate test of quantum electrodynamics (QED) in any highly charged ion on the Li-like (quasi-hydrogenic) isoelectronic sequence. These results provide a more stringent test of QED than even the most accurate results available for hydrogenic uranium, and demonstrate that research activity focused on Tokamak diagnostics can have important spin-off benefits for fundamental science.

Our previously published paper on the Na-like D-lines cited in the previous paragraph also contains a review of all the data available on the isoelectronic sequence, and points out good agreement between theory and experiment, except near $Z=54$ where data from three independent groups, on several types of light sources, agree with each other, but disagree with theory. When considered together, the statistical significance of this discrepancy is very high (4-sigma). Consequently, we have performed a more detailed analysis of Xe data taken at a variety of electron beam energies, and presented evidence for a previously unaccounted for systematic effect. A section on these findings is included in a paper that was recently accepted for publication.

We also completed analysis and identification of EUV spectra from the $3 d^{n}$ ions of tungsten. We found that these spectra between 10 and $20 \mathrm{~nm}$ contain only forbidden magnetic-dipole lines that can be used for plasma diagnostics in hot fusion devices including ITER.

Three other papers that were in progress last year (discussed in our previous progress report) have been published or accepted for publication.

Our current spectroscopy efforts using the EBIT are closely coupled to our modeling expertise, the spectral analysis expertise of our senior spectroscopists, and our data compilation activities (see other sections of this report for details).

On the hardware and human resources fronts, a new higher resolution and lower noise 
EUV CCD detector was procured and bench tested, and a new postdoctoral associate was trained (D. Osin, from Y. Maron's plasma group at the Weizmann Institute of Science). Our former postdoctoral associate (I.N. Draganic), has moved to the Oak Ridge.

\section{Publications}

1. J. D. Gillaspy, C. T. Chantler, D. Paterson, L. T. Hudson, F. G. Serpa, and E. Takacs, "First measurement of lyman alpha $\mathrm{X}$-ray lines in hydrogen-like vanadium: results and implications for precision wavelength metrology and tests of QED", Journal of Physics B 43, 074021 (2010).

2. E. Silver, N. S. Brickhouse, T. Lin, G. X. Chen, K. Kirby, J. D. Gillaspy, J. N. Tan, and J. M. Laming, "X-ray spectroscopy of highly charged ions in laboratory and astrophysical plasmas", in Recent advances in spectroscopy: theoretical, astrophysical, and experimental perspectives, edited by R. K. Chaudhuri et al. (Springer, Berlin, 2010), p. 1 (2010).

3. W. L. Wiese, M. A. Ali, I. Draganic, J. R. Fuhr, J. D. Gillaspy, R. Ibacache, D. E. Kelleher, A. E. Kramida, L. Podobedova, Yu. Ralchenko, J. Reader, E. B. Saloman, P. M. Stone, "Spectral properties of heavy elements of fusion interest", Atomic and Plasma-Material Interaction Data for Fusion, (in press, 2010).

4. J. D. Gillaspy, "Testing QED in sodium-like gold and xenon: using atomic spectroscopy and an EBIT to probe the quantum vacuum", Journal of Instrumentation, (in press, 2010).

5. E. S. Silver, J. D. Gillaspy, P. Gokhale, E. P. Kanter, N. S. Brickhouse, R. W. Dunford, K. Kirby, T. Lin, J. Mcdonald, D. Schneider, S. Seifert, L. Young, "Towards experimental evidence of hard X-ray photoionization of highly charged krypton", Application of Accelerators in Research and Industry (under review, 2010).

6. J. D. Gillaspy, T. Lin, L. Tedesco, J. N. Tan, J. M. Pomeroy, J. M. Laming, N. Brickhouse, G.-X. Chen, and E. Silver, "Fe XVII x-ray line ratios for accurate astrophysical plasma diagnostics", Astrophysical Journal (under review, 2010).

7. I. Draganic, Yu. Ralchenko, J. Reader, J. D. Gillaspy, J. N. Tan, J. M. Pomeroy, S. M. Brewer, D. Osin, "EUV spectral lines of highly-charged Hf, Ta, and Au ions observed with an electron beam ion trap", Journal of Physics B (submitted, 2010).

Talks

Spectra we acquired with an EBIT were presented in the following invited talks and poster presentation, as well as in additional talks given by J. Reader, Yu. Ralchenko, A. Kramida, and J. Curry (see other sections of this report). 
1. "Progress Report on NIST Work" J. D. Gillaspy, 11th International Conference on Electron Beam Ion Sources and Traps, Stockholm, Sweden, April 2010.

2. "New Opportunities for Measurement Science at NIST", W. Ott, Department of Physics, University of Melbourne, Melbourne, Australia, August, 2010.

3. "Electron Beam Ion Trap and Plasma Physics", J. D Gillaspy, talk to the National Academies Panel on Physics, NIST, Gaithersburg, MD, February, 2010.

4. "First Deployment of and Electron Beam Ion Trap at an Advanced Source of Hard Xrays", J. D. Gillaspy et al., APS April Meeting, Washington, DC, February 2010 (poster).

5. "Using Microcalorimeters to Study Highly Charged Ions", E. Silver, 21st International Conference on Applications of Accelerators in Research and Industry, Fort Worth, TX, August 2010.

6. "Spectroscopy and atomic physics of highly charged ions observed with an Electron Beam Ion Trap at NIST," J. Reader, Institute of Spectroscpy, Moscow, Russia, September 2010.

\section{Atomic Structure Calculations and Modeling of Plasma Spectra (Yu. Ralchenko and C.F. Fischer)}

C. Froese Fischer actively worked on development of new multiconfiguration nonrelativistic and relativistic methods for calculation of atomic structure parameters. She published several papers addressing (i) new computational procedure combining the most accurate MCHF and B-spline BSR codes for calculation of energy levels, radiative transition probabilities and photoionization cross sections, (ii) accurate transition data for important $3 p^{2}, 3 p^{3}$ and $3 p^{4}$ ions of $\mathrm{Fe}, \mathrm{Co}$ and $\mathrm{Ni}$, and (iii) accurate account of correlation effects in complex ions through biorthonormal transformations. This research provides new ideas and techniques for accurate calculations for fusion-related atomic systems.

Yu. Ralchenko's research was devoted to the collisional-radiative (CR) modeling of hot plasmas of EBITs and tokamaks. He used CR simulations to perform identification of the spectral lines from highly-charged high-Z ions measured on the NIST EBIT. A manuscript on the newly measured EUV lines from $\mathrm{Hf}, \mathrm{Ta}$ and $\mathrm{Au}$ has been completed.

In collaboration with the TEXTOR group in Germany, he applied a new parabolic-state CR model of a neutral beam to the analysis of the motional Stark effect (MSE) in tokamaks. A large number of proton-impact cross sections were calculated using an original double-rotation method applied to the eikonal approximation. This work was able to explain the 1993 measurements from the JET tokamak in UK. 
Yu. Ralchenko also organized the $7^{\text {th }}$ Non-LTE Code Comparison Workshop (December 2009 , Athens, Greece) where CR model developers from various countries, including representative from LLNL, LANL and Sandia National Laboratory, gathered to compare their $\mathrm{CR}$ codes. One of the cases offered for comparisons included tungsten spectra at typical conditions of ITER. The comparison results will be important for improvement of $\mathrm{CR}$ models used in tokamak plasma kinetic simulations.

Finally, Yu. Ralchenko was the Editor of the Special Issue of Journal of Physics B on spectroscopic diagnostics of magnetic fusion plasmas. This compendium of papers from the leading MCF groups will serve as a valuable resource for the community.

\section{Publications on atomic structure}

1. T. Carette, C. F. Fischer, et al., "Isotope shift in the sulfur electron affinity: Observation and theory," Phys. Rev. A 81, 042522 (2010).

2. S. Verdebout, C. F. Fischer et al., "Exploring biorthonormal transformations of pair-correlation functions in atomic structure variational calculations," J. Phys. B 43, 074017 (2010).

3. C. Froese Fischer, "Towards accurate transition data for $3 p^{2}, 3 p^{3}$ and $3 p^{4}$ levels of Fe, Co and Ni ions," J. Phys. B 43, 074020 (2010).

4. O. Zatsarinny and C. Froese Fischer, "Atomic Structure Calculations using MCHF and BSR," Comp. Phys. Comm. 180, 2041 (2009).

\section{Publications on modeling of plasma spectra}

1. U. Zastrau, Yu. Ralchenko, et al., "Temperature and $K \alpha$-yield radial distributions in laser-produced solid-density plasmas imaged with ultrahigh-resolution $\mathrm{X}$-ray spectroscopy," Phys. Rev. E 81, 026406 (2010).

2. O.Marchuk, Yu. Ralchenko, R. K. Janev, W. Biel, E. Delabie and A.M. Urnov, "Collisional Excitation and Emission of $\mathrm{H}_{\alpha}$ Stark Multiplet in Fusion Plasmas," J. Phys. B 43, 011002 (2010).

3. U. Feldman, Yu. Ralchenko, and G.A. Doschek, "The Effect of Hot Coronal Electrons on Extreme-Ultraviolet Spectral Lines of He II Emitted by Solar Transition Region Plasmas," Astrophys. J., 708, 244 (2010). 